Article

\title{
Impact of Input Filtering and Architecture Selection Strategies on GRU Runoff Forecasting: A Case Study in the Wei River Basin, Shaanxi, China
}

\author{
Qianyang Wang, Yuan Liu, Qimeng Yue, Yuexin Zheng, Xiaolei Yao and Jingshan Yu * \\ College of Water Sciences, Beijing Normal University, Beijing 100875, China; \\ 201931470001@mail.bnu.edu.cn (Q.W.); 201921470018@mail.bnu.edu.cn (Y.L.); yqm2012@yeah.net (Q.Y.); \\ hss_zhengyuexin@126.com (Y.Z.); 11312020021@bnu.edu.cn (X.Y.) \\ * Correspondence: jingshan@bnu.edu.cn; Tel.: +86-10-5880-7814
}

Received: 21 November 2020; Accepted: 15 December 2020; Published: 16 December 2020

\begin{abstract}
A gated recurrent unit (GRU) network, which is a kind of artificial neural network (ANN), has been increasingly applied to runoff forecasting. However, knowledge about the impact of different input data filtering strategies and the implications of different architectures on the GRU runoff forecasting model's performance is still insufficient. This study has selected the daily rainfall and runoff data from 2007 to 2014 in the Wei River basin in Shaanxi, China, and assessed six different scenarios to explore the patterns of that impact. In the scenarios, four manually-selected rainfall or runoff data combinations and principal component analysis (PCA) denoised input have been considered along with single directional and bi-directional GRU network architectures. The performance has been evaluated from the aspect of robustness to 48 various hypermeter combinations, also, optimized accuracy in one-day-ahead $(\mathrm{T}+1)$ and two-day-ahead $(\mathrm{T}+2)$ forecasting for the overall forecasting process and the flood peak forecasts. The results suggest that the rainfall data can enhance the robustness of the model, especially in $\mathrm{T}+2$ forecasting. Additionally, it slightly introduces noise and affects the optimized prediction accuracy in $\mathrm{T}+1$ forecasting, but significantly improves the accuracy in $\mathrm{T}+2$ forecasting. Though with relevance $(\mathrm{R}=0.409 \sim 0.763$, Grey correlation grade $>0.99)$, the runoff data at the adjacent tributary has an adverse effect on the robustness, but can enhance the accuracy of the flood peak forecasts with a short lead time. The models with PCA denoised input has an equivalent, even better performance on the robustness and accuracy compared with the models with the well manually filtered data; though slightly reduces the time-step robustness, the bi-directional architecture can enhance the prediction accuracy. All the scenarios provide acceptable forecasting results (NSE of 0.927 0.951 for $\mathrm{T}+1$ forecasting and $0.745 \sim 0.836$ for $\mathrm{T}+2$ forecasting) when the hyperparameters have already been optimized. Based on the results, recommendations have been provided for the construction of the GRU runoff forecasting model.
\end{abstract}

Keywords: ANN; GRU; runoff forecasting; input filtering; architecture

\section{Introduction}

Runoff forecasting plays an essential role in flood mitigation management, agricultural water management, water transportations, and other socio-economical activities closely related to water resources. Many approaches, including physics-based models [1-3] and data-driven models [4-6], have been developed during recent decades to obtain a precise prediction result of the runoff.

Some physics-based hydrological models, such as SWAT [7-9], TOPMODEL/BTOP-MC [10,11], and MIKE SHE [12,13], have already been widely used to simulate the rainfall-runoff relationship for runoff prediction because of the definite and explainable mechanisms and the theoretically high 
accuracy of the modelling results, in addition to the user-friendly interfaces of the modelling software. However, the above-mentioned physics-based models usually require the observed rainfall as the input and the observed runoff data for calibration/validation and some additional data, like the digital elevation model (DEM), soil types, and land use information. The modelling results usually show the different extent of dependencies on the presence, appropriateness, and the resolution of those additional data [14]. This dependency might restrain the practical implementation of the models.

Contrary to the hydrological/hydrodynamic models that are mainly based upon a series of definite physical mechanisms, the data-driven models only consider the statistical relationship of the data [15]. Machine learning is one of the most commonly utilized approaches for exploring the numeric connections between the input and the target data. Several kinds of machine learning model that can be used to attain a precise estimation on the short-term runoff have been shown in several pieces of research. For example, the support vector machine [16-18] and the random forest regressor [19,20]. As a subset of machine learning, deep learning, which is mainly represented by the artificial neural network (ANN) techniques, is of great interest nowadays due to the booming computer science and algorithms [21]. Since its ability to seek out the complicated nonlinear relationships between the given datasets, the ANNs can be applied to complex systems' modeling tasks. In the hydrological field, the ANNs have been used for different aims, for instance, flood or runoff forecasting [17,22-24], rainfall forecasting [25-27], and evapotranspiration prediction [28,29].

The ANNs can also be categorized into several subdivisions according to their specific structures; in other words, their characteristics. The designs of the ANNs result in the diversity of their advancements and limitations [30]. For example, the convolutional neural networks have the kernel to recognize the spatial distribution pattern of data; consequently, it has been implemented commonly for imagery classification [31-33], however, the existence of pooling layers may cause the loss of abundant information [34]. The recurrent neural network (RNN) is a "loop" structure that has a state to store the information from the previous steps; thus, it can deal with the time-series data that has time dependency. Despite the advancement of the RNNs from the aspect of time series processing, when dealing with the long-term dependency sequences, the performance of the conventional RNN cells is not satisfactory enough as a result of the vanishing or exploding gradients. Long short-term memory (LSTM) networks, a variant of RNNs [35], solved the drawbacks of the traditional RNNs. There has been plenty of research in various realms that utilized the LSTM networks to process the time series data successfully, for instance, natural language processing (NLP) [36-38], water or air quality forecasting [39-41], and disease diagnoses [42,43]. The LSTM network also has some variants; one is the gated recurrent unit (GRU) network [44], which needs fewer calculations than an LSTM cell. As a result, the GRU networks usually show a higher training efficiency than the LSTM networks $[45,46]$.

For the runoff forecasting, the runoff has an apparent long-term dependency on the rainfall data [47] and the reliance on the previous upstream runoff data; the characteristics of LSTM/GRU are appropriate for this type of assignment. Several studies have shown that the LTSM networks or GRU networks can obtain impressive results when carrying out runoff or flood forecasting assignments. The LSTM network has been implemented in the Da River basin in Vietnam for one-day, two-day, and three-day ahead flowrate forecasting cases and got the Nash-Sutcliffe efficiency (NSE) of 99\%, 95\%, and $87 \%$ correspondingly for each case [15]. A flood forecasting model with a spatiotemporal attention mechanism based on LSTM has been used in Lech and Changhua river basins and obtained a lower root mean square error (RMSE), a lower mean absolute percent error (MAPE), and a higher deterministic coefficient (DC) compared with SVM and fully-connected network (FCN) for six- and nine-time step flood predictions [48]. A self-attentive long short-term memory (SA-LSTM) network has been evaluated at eight runoff datasets for 1 7 days ahead forecasting. It has been shown to have the best prediction accuracy in several models [49]. A deep neural network called CAGANet, which consists of a convolutional layer, an attention mechanism, a GRU neural network, and an autoregressive (AR) model, has been proposed for daily runoff predictions in Qingxi river basin, Sichuan Province, China, the NSE reached 0.993 [50]. Despite the good performances of LSTM networks and GRU networks 
in recent studies, most of them focus on improving the optimized prediction accuracy by modifying or coupling the models. There have been few attempts to demonstrate the impact of different input datasets, as well as the model's structure on the performance of the model, especially in the model construction stage. Though not mainly focusing on this problem, some researchers have preliminarily revealed that input filtering has an impact on the forecasting model. For instance, relevant research [15] suggests that a higher correlation coefficient between the input and the target datasets can better the forecasting model's performance. Meanwhile, the inclusion of the rainfall data might not significantly enhance the prediction accuracy and sometimes even negatively impact it. Another study [51] shows that a better prediction result (the test NSE of 0.942 compared with 0.666 ) has been obtained when using a selected subset of all rain gauges as the input. Furthermore, the LSTM or GRU networks' architectures can also influence the forecasting results [52,53].

The problem is that although these studies have shown the impact of the input and the networks' structure on the final performance, this impact still needs to be adequately evaluated. It should be carefully considered at the stage of preprocessing and model construction.

In this research, to comprehensively explore the specific impact of the different input filtering strategies, in addition to the effects of the networks' architectures on the GRU-based runoff forecasting model, we constructed several models that use the flowrate (at hydrological stations) and the rainfall data (at meteorological stations) as the input. The Wei River basin, which is a typical region with a continental climate and distinct seasons [54], located in Shaanxi, China, is selected as the study area. Four scenarios of the input dataset with different combinations of the hydrological stations and the meteorological stations, two principal component analysis (PCA) denoising based scenarios with single- and bi-directional architectures for one-day-ahead and two-day-ahead predictions have been conducted. A scope of hyperparameters, including the number of hidden units, the number of hidden layers, and the length of the input time steps, has been set to make the comparison. The models have been evaluated from the aspect of robustness [55] to hyperparameters and accuracy. The knowledge about the particular impact of the input and architectures on the performance of the model can be valuable in the model construction stage since it can act as guidance and help to construct a more robust and accurate forecasting model within a more convenient process. Recommendations based on that knowledge could be a good reference for future studies and practical applications.

\section{Methodology}

\subsection{Preprocessing}

\subsubsection{Principal Component Analysis (PCA) Denoising}

The PCA method is utilized in the preprocessing stage in two of the scenarios (refer to the scenarios setting situation in Section 3.2) for column vector selection. Typically, it is used as an unsupervised algorithm to delete the redundant components in the input dataset and only maintain the components that explain most of the variance. Through the PCA operation, the initial N-dimensional matrix will be transformed into a K-dimensional matrix $(\mathrm{K}<\mathrm{N})$. The calculation processes in the PCA component reduction operation can refer to the following literature [56-58].

After the component reduction process in the PCA operation, the processed matrix is then re-projected to form a matrix that has the same dimension (or the same shape) as the initial input matrix. What should be mentioned is that the filtered information will not be included in the re-projection process. 


\subsubsection{Normalization}

For the scenarios without PCA denoising operation, the rainfall and runoff data is normalized with Equation (1), called Min-Max normalization. The normalized data has a value range of [1]:

$$
x^{\prime}=\frac{x-\min (x)}{\max (x)-\min (x)}
$$

where $x^{\prime}$ is the normalized data, $\min (x)$ and $\max (x)$ are the minimum and maximum values of each column in the input dataset.

For the PCA denoising operation scenarios, the data is normalized with Equation (2), called Max-Abs normalization. The normalization result has a value range of $[-1,1]$ :

$$
x^{\prime}=\frac{x}{|\max (x)|}
$$

where $x^{\prime}$ is the normalized data, $\max (x)$ is the maximum value of each column in the input dataset.

\subsubsection{Sliding Window Sampling}

A raw input dataset with $\mathrm{M}$ rows and $\mathrm{N}+1$ columns means that it has $\mathrm{M}$ records; each has $\mathrm{N}$ different features and a prediction target. For the GRU network, since the input should be a sequence with multiple time steps, a sliding window method has been utilized to generate samples. Take the time step (sliding window size) $=3$ as an example. The data structure, in addition to the sampling process, is shown in Figure 1. For the input dataset that without a PCA denoising operation, the column index $F_{n}$ represents the hydrological stations or the meteorological stations. For the one with a PCA denoising operation, the column index $F_{n}$ represents a feature. The row index $t_{n}$ means time, which has a unit of day in this research. The $Y$ is the column of the prediction target values.

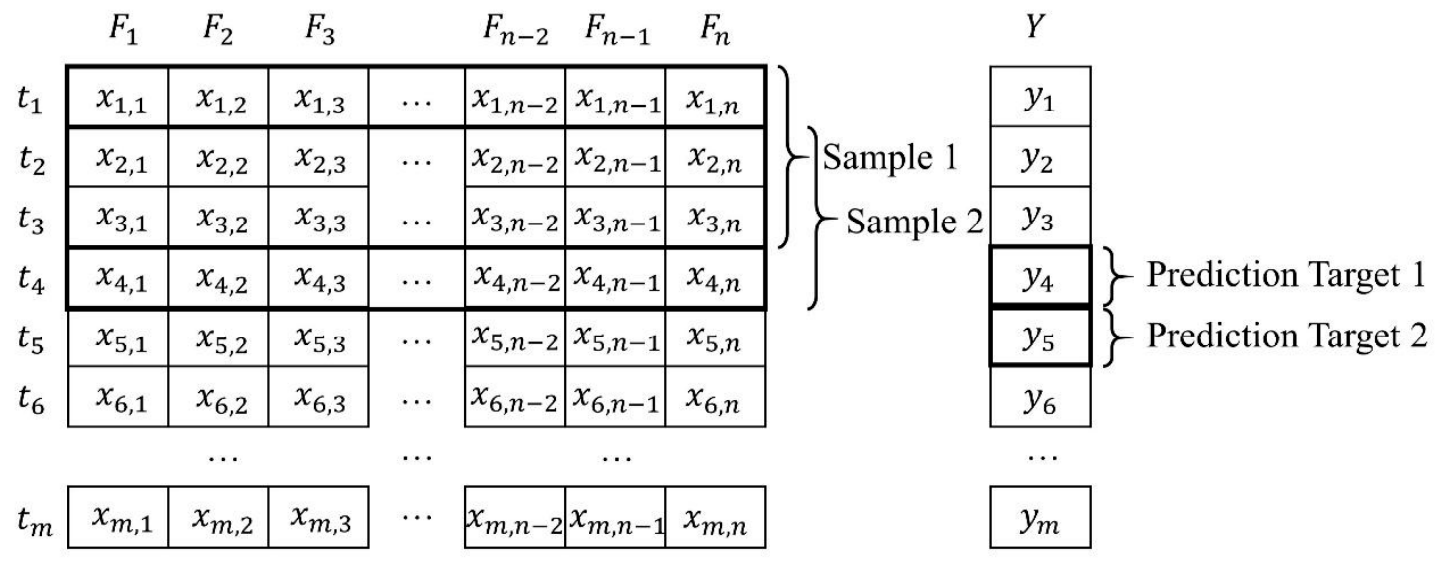

Figure 1. An example of the sliding window method, $F$ denotes the feature column, and $t$ denotes a record (or sample).

\subsection{Gated Recurrent Unit (GRU)}

The GRU cell [44] contains two gate units (three gates in some of the literature [59], which include the output gate): the update gate and the reset gate. The internal structure of a primary GRU cell is shown in Figure 2. The unimportant information in the hidden state from the previous time step can be discarded through the reset gate. In contrast, useful information will be retained to affect the current hidden state. The whole forward propagation steps are as shown in Equations (3)-(6):

$$
r_{t}=\sigma\left(W_{r} \cdot\left[h_{t-1}, x_{t}\right]\right),
$$




$$
\begin{gathered}
z_{t}=\sigma\left(W_{z} \cdot\left[h_{t-1}, x_{t}\right]\right), \\
\widetilde{h}_{t}=\tanh \left(W_{\tilde{h}} \cdot\left[r_{t} \odot h_{t-1}, x_{t}\right]\right), \\
h_{t}=\left(1-z_{t}\right) \odot h_{t-1}+z_{t} \odot \widetilde{h}_{t} .
\end{gathered}
$$

where $r_{t}$ and $z_{t}$ denote the output of the reset gate and the update gate, respectively. $\sigma$ represents the sigmoid activation function. $\odot$ represents the Hadamard product (element-wise product). $[a, b]$ denotes the concatenate operation for two vectors. $h_{t}$ represents the current hidden state.

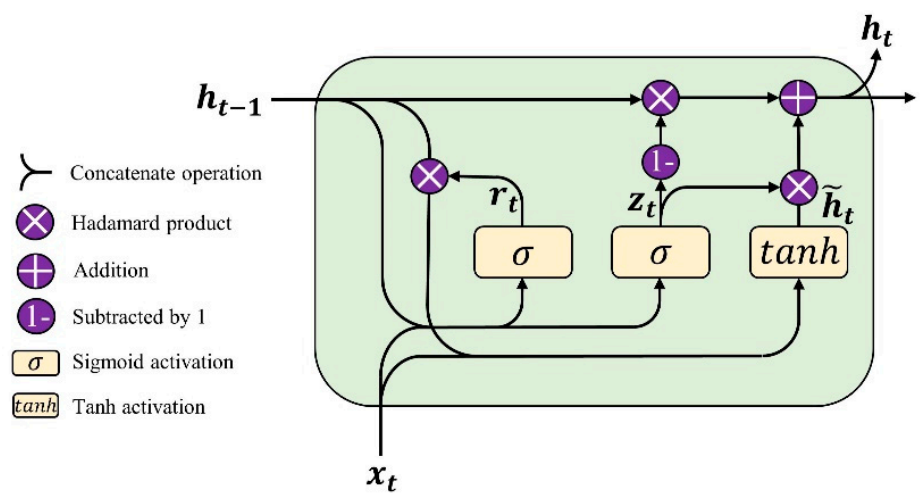

Figure 2. The structure of a basic GRU cell.

\subsection{Stacked and Bi-Directional GRU (bi-GRU)}

The combination of several layers of the basic LSTM or GRU cells may enhance the model's ability when learning the nonlinear relationships and provide a better prediction accuracy $[53,60]$. The typical structure of a stacked GRU network with two layers is shown in Figure 3. The first layer's hidden state is utilized as the input of the second layer for further calculations. The result could be obtained from the output of the second layer.

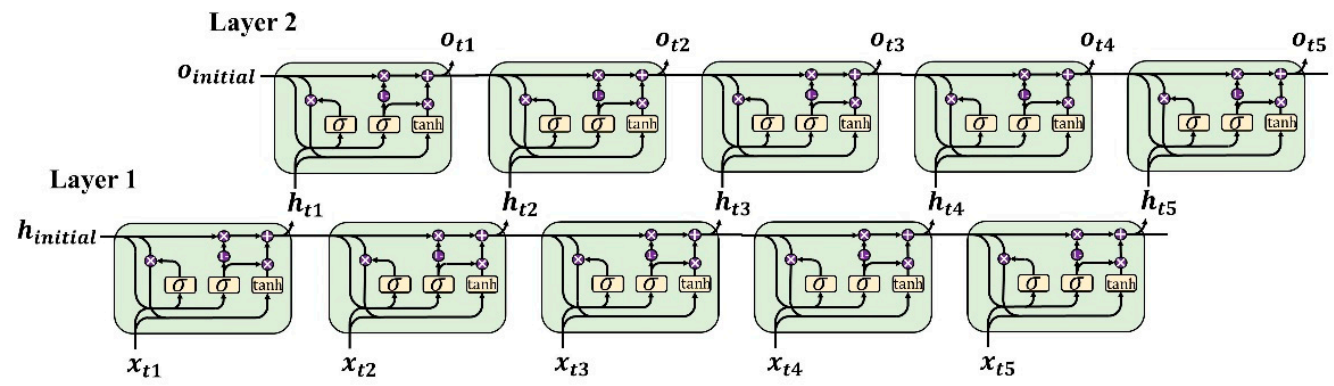

Figure 3. The typical structure of a double layer stacked GRU network, the second layer obtains the state of the first layer for further calculation.

A basic bi-GRU network (Figure 4) has two single layers that propagate in inverse directions. The forward layer uses the first record in the input sequence as the beginning, while the backward layer uses the last record as its beginning. The overall state is the summation of the states from both of the layers; the final state can also be covered by an activation layer. 


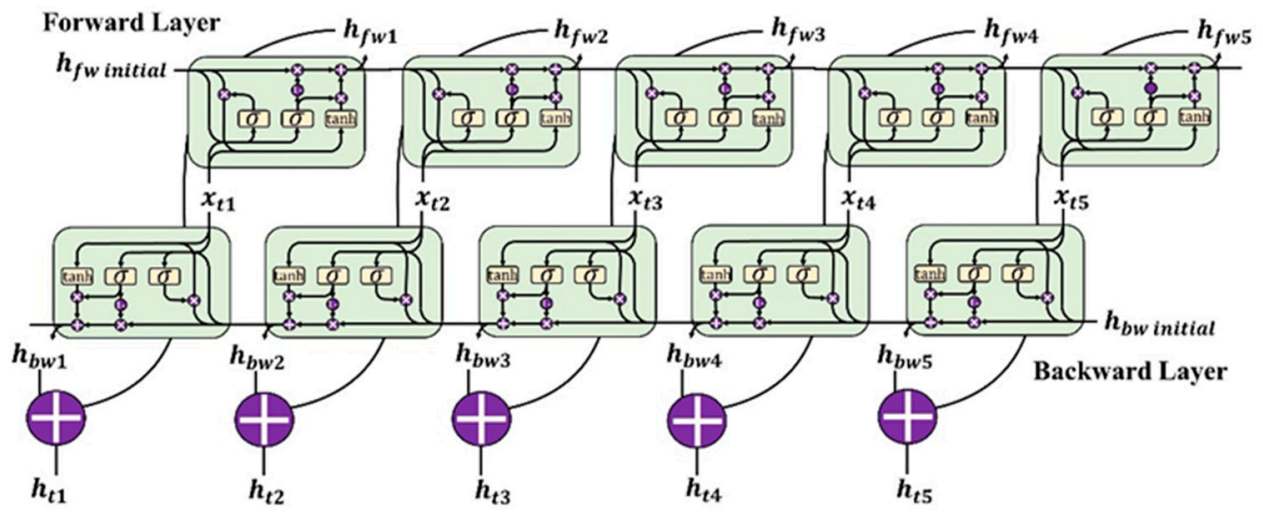

Figure 4. The typical structure of a bi-GRU network with two single layers.

\subsection{Model Evaluation}

Nash-Sutcliffe efficiency (NSE), root mean square error (RMSE), and mean absolute error (MAE) has been used in each simulation, as shown in Equations (7)-(9), respectively:

$$
\begin{gathered}
N S E=1-\frac{\sum_{i=1}^{n}\left(O_{i}-P_{i}\right)^{2}}{\sum_{i=1}^{n}\left(O_{i}-\bar{O}\right)^{2}}, \\
R M S E=\sqrt{\frac{1}{n} \sum_{i=1}^{n}\left(O_{i}-P_{i}\right)^{2},} \\
M A E=\frac{1}{n} \times \sum_{i=1}^{n}\left|O_{i}-P_{i}\right| .
\end{gathered}
$$

where $O_{i}$ is the observed flowrate, $P_{i}$ is the predicted flowrate, $\bar{O}$ is the mean value of the observed flowrate, $\mathrm{n}$ is the total number of observations. The NSE values range from $-\infty$ to 1 . A higher NSE value indicates a better fitting result. Both the RMSE and MAE ranges from 0 to $+\infty$ (with a unit of $\mathrm{m}^{3} / \mathrm{s}$ in this study), the lower the RMSE and MAE are, the better the model performs. The RMSE is more sensitive to abnormal values than the MAE.

For the robustness evaluation, the mean standard deviation of the abovementioned metrics along the time steps (one of the hyperparameter, that is, the length of input sequence) will be calculated through Equation (10) metrics as mentioned earlier in each scenario:

$$
\sigma=\sqrt{\frac{1}{n} \sum_{i=1}^{n}\left(x_{i}-\mu_{i}\right)^{2}} .
$$

where $\sigma$ is the standard deviation, $x_{i}$ is the $i$ th metric, $\mu_{i}$ is the mean value of the metrics. The value of standard deviation is greater than 0 , a lower standard deviation indicates that the metrics are more aggregated and more robust to time steps.

For the accuracy evaluation, besides the NSE, RMSE, and MAE used in the overall evaluation, the relative error (as shown in Equation (11)) has been used to evaluate the performance on flood peak forecasts:

$$
\delta=\frac{\left|O_{\text {peak }}-P_{\text {peak }}\right|}{O_{\text {peak }}} \cdot 100 \%
$$

where $\delta$ is the relative error, $O_{\text {peak }}$ is the observed peak flow, $P_{\text {peak }}$ is the predicted value of the observed peak flow. The relative error value ranges from 0 to $+\infty$, the closer the value is to 0 , the better the fitting is. 


\section{Case Study and Materials}

\subsection{Research Area and Data}

The Wei River basin $\left(33^{\circ} \mathrm{N} \sim 37^{\circ} \mathrm{N}, 104^{\circ} \mathrm{E} \sim 110^{\circ} \mathrm{E}\right)$, which is mainly located in Shaanxi Province, China, has an area of 135.9 thousand square kilometers. It is a representative region in the western and northern parts of China. Located in a semi-humid to semi-arid warm temperature climate zone, the basin has a continental monsoon climate [61], and the elevation ranges from $324 \mathrm{~m}$ to $3911 \mathrm{~m}$. The downstream region has annual precipitation ranges from $600 \sim 800 \mathrm{~mm}$, while the annual precipitation at the upstream region ranges from $350 \sim 500 \mathrm{~mm}$. The period from June to October accounts for $65 \sim 80 \%$ of the total annual precipitation. As a result, the downstream area is affected by flood events frequently. The basin accounts for $64 \%$ of the total population, $72 \%$ of the irrigation area, and $80 \%$ of the GDP in Shaanxi Province from socio-economical activities. Additionally, this basin is located at the Loess Plateau, which is very sensitive to soil erosion caused by rainfall-runoff processes. Hence, stable and reliable runoff forecasting can have high merit for flood warnings, water resources management, and water and soil conservation in this region. The Wei River is the largest tributary of the Huang River containing the main channel and two main branches (the Jing River and the Beiluo River).

The daily runoff data (gotten from the Hydrological Yearbook of the People's Republic of China) at 14 hydrological stations and the daily rainfall data (gotten from China Meteorological Data Service Center, http://data.cma.cn) at 10 meteorological stations from 2007 to 2014 were collected. Good continuity makes the collected data suitable for the sequential learning model. As a result of the good sufficiency of stations, a vector selection process becomes necessary, the impact of several input combinations can be explored. The research area, the locations of the hydrological and meteorological stations, and the prediction target station are shown in Figure 5. The summaries of the data at the hydrological stations and the meteorological stations are given in Tables 1 and 2, respectively. The grey relation grade [62] and the Pearson correlation coefficient between each station and the prediction target, Huaxian station, have been calculated to show the data's relevance.

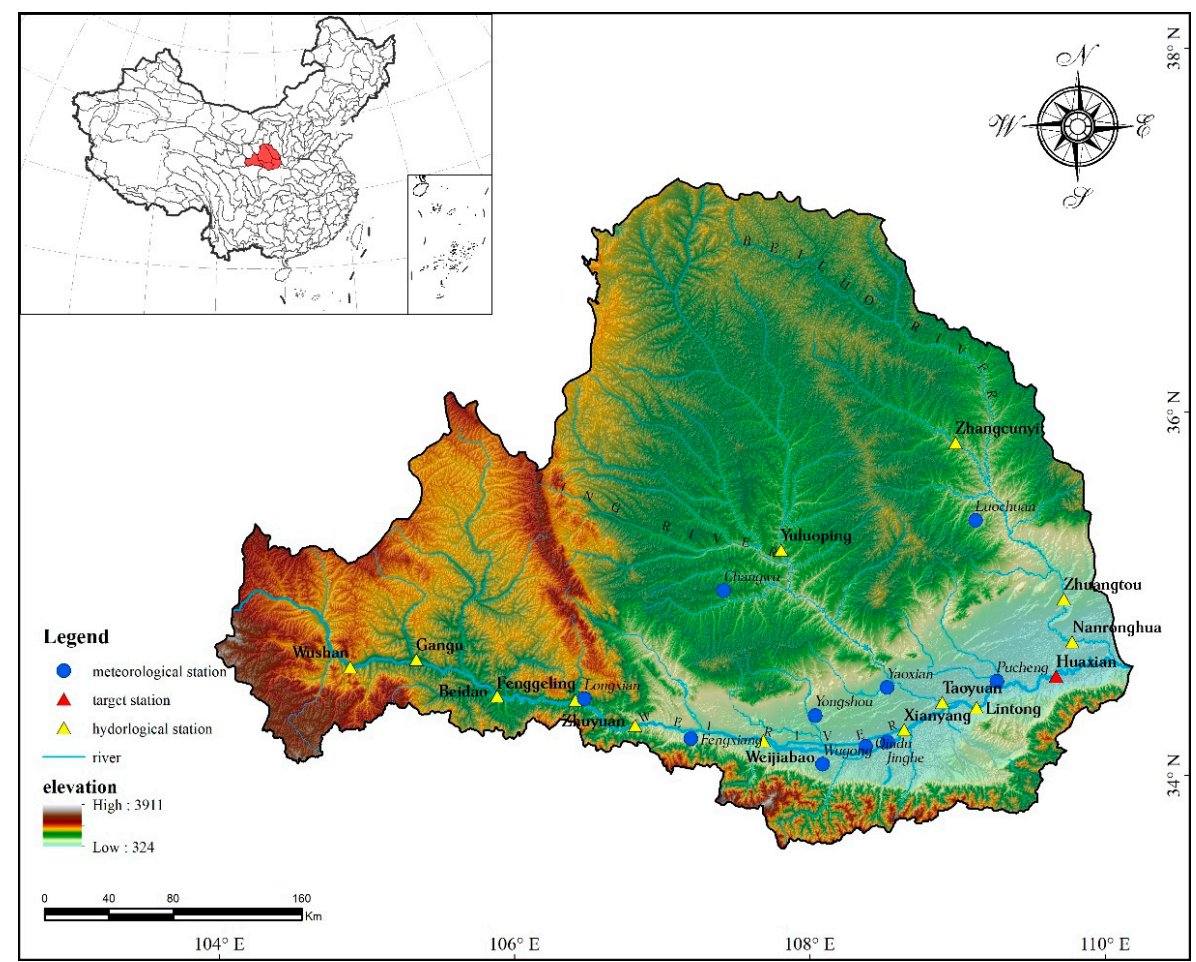

Figure 5. The research area with the locations of hydrological and meteorological stations, in addition to the prediction target. The whole basin can be divided into three main sub-basins, which are the main channel basin of the Wei River, the Jing River basin, and the Beiluo River basin. 
Table 1. A summary of the runoff data at hydrological stations.

\begin{tabular}{ccccccc}
\hline Stations & Sub-Basin & $\begin{array}{c}\text { Distance } \\
(\mathbf{K m})\end{array}$ & Max $\left(\mathbf{m}^{\mathbf{3}} \mathbf{s}\right)$ & Mean $\left(\mathbf{m}^{\mathbf{3}} / \mathbf{s}\right)$ & $\begin{array}{c}\text { Grey } \\
\text { Relation }\end{array}$ & $\mathbf{R}$ \\
\hline Huaxian & W & 0.0 & 4410.0 & 171.7 & 1.000 & 1.000 \\
Nanronghua & B.L. & 23.5 & 250.0 & 12.5 & 0.993 & 0.763 \\
Zhuangtou & B.L. & 49.4 & 440.0 & 15.5 & 0.993 & 0.757 \\
Lintong & W & 53.3 & 4570.0 & 182.0 & 0.996 & 0.888 \\
Taoyuan & J & 72.8 & 906.0 & 32.7 & 0.993 & 0.703 \\
Xianyang & W & 100.3 & 2890.0 & 94.9 & 0.995 & 0.809 \\
Zhangcunyi & B.L. & 158.5 & 147.0 & 2.8 & 0.990 & 0.409 \\
Weijiabao & W & 186.7 & 1830.0 & 64.9 & 0.992 & 0.727 \\
Yuluoping & J & 188.8 & 687.0 & 10.1 & 0.990 & 0.194 \\
Zhuyuan & W & 263.9 & 59.5 & 2.0 & 0.992 & 0.536 \\
Fenggeling & W & 299.8 & 114.0 & 3.7 & 0.993 & 0.545 \\
Beidao & W & 348.2 & 959.0 & 22.8 & 0.991 & 0.365 \\
Gangu & W & 398.5 & 90.7 & 0.6 & 0.987 & 0.098 \\
Wushan & W & 439.6 & 699.0 & 12.1 & 0.991 & 0.274 \\
\hline
\end{tabular}

$\mathrm{W}=$ the Wei River, B.L. = the Beiluo River, $\mathrm{J}=$ the Jing River.

Table 2. A summary of the rainfall data at meteorological stations.

\begin{tabular}{ccccccc}
\hline Stations & Sub-Basin & $\begin{array}{c}\text { Distance } \\
\mathbf{( K m})\end{array}$ & Max $(\mathbf{m m})$ & Mean $(\mathbf{m m})$ & $\begin{array}{c}\text { Grey } \\
\text { Relation }\end{array}$ & R \\
\hline Pucheng & W & 37.3 & 60.8 & 1.4 & 0.979 & 0.114 \\
Yaoxian & W & 105.6 & 69.0 & 1.6 & 0.979 & 0.120 \\
Luochuan & B.L. & 109.1 & 107.5 & 1.7 & 0.979 & 0.096 \\
Jinghe & W & 115.2 & 117.3 & 1.5 & 0.979 & 0.109 \\
Qindu & W & 126.3 & 158.5 & 1.5 & 0.979 & 0.093 \\
Yongshou & W & 151.9 & 100.1 & 1.6 & 0.979 & 0.106 \\
Wugong & W & 155.3 & 101.4 & 1.7 & 0.979 & 0.124 \\
Changwu & J & 213.7 & 142.2 & 1.6 & 0.980 & 0.089 \\
Fengxiang & W & 232.6 & 76.2 & 1.7 & 0.979 & 0.097 \\
Longxian & W & 296.9 & 214.6 & 1.6 & 0.980 & 0.094 \\
\hline
\end{tabular}

$\mathrm{W}=$ the Wei River, B.L. = the Beiluo River, $\mathrm{J}=$ the Jing River.

\subsection{Scenarios and Traning Process}

As shown in Table 3, six scenarios for both the one-day-ahead and two-day-ahead forecasting have been set to evaluate the impact of different input filtering and model construction strategies. Referring to Figure 5 and Table 1 in Section 3.1, though the Beiluo River is a tributary flowing downstream of the prediction target, the distance from each of the hydrological stations in the Beiluo River basin is relatively close (23.5 km from Nanronghua station, $49.4 \mathrm{~km}$ from Zhuangtou station, $158.5 \mathrm{~km}$ from Zhangcunyi station). The grey relation analysis and the Pearson correlation analysis show that the data in the Beiluo river basin is also moderately or highly relevant $(R=0.763,0.757$, and 0.409; grey relation grades over 0.99) to the data at the prediction target. Thus, the runoff data from the Beiluo River may be valuable as a reference and should be cautiously evaluated.

Table 3. Summary of the setting condition of scenarios.

\begin{tabular}{cccccc}
\hline $\begin{array}{c}\text { Prediction } \\
\text { Target }\end{array}$ & Scenario & RNN Cell & Pre-Processing & $\begin{array}{c}\text { Hydrological } \\
\text { Stations } \\
\text { (Runoff Data) }\end{array}$ & $\begin{array}{c}\text { Meteorological } \\
\text { Stations } \\
\text { (Rainfall Data) }\end{array}$ \\
\hline & S1 & GRU & MMN & all included & all included \\
& S2 & GRU & MMN & all included & all excluded \\
T +1 & S3 & GRU & MMN & B.L. excluded & all included \\
& S4 & GRU & MMN & B.L. excluded & all excluded \\
& S5 & GRU & PCA + MAN & all included & all included \\
& S6 & bi-GRU & PCA + MAN & all included & all included \\
\hline
\end{tabular}


Table 3. Cont.

\begin{tabular}{cccccc}
\hline $\begin{array}{c}\text { Prediction } \\
\text { Target }\end{array}$ & Scenario & RNN Cell & Pre-Processing & $\begin{array}{c}\text { Hydrological } \\
\text { Stations } \\
\text { (Runoff Data) }\end{array}$ & $\begin{array}{c}\text { Meteorological } \\
\text { Stations } \\
\text { (Rainfall Data) }\end{array}$ \\
\hline \multirow{4}{*}{$\mathrm{T}+2$} & $\mathrm{~S} 1$ & GRU & MMN & all included & all included \\
& $\mathrm{S} 2$ & GRU & MMN & all included & all excluded \\
& S3 & GRU & MMN & B.L. excluded & all included \\
& S4 & GRU & MMN & B.L. excluded & all excluded \\
& S5 & GRU & PCA + MAN & all included & all included \\
& S6 & bi-GRU & PCA + MAN & all included & all included \\
\hline
\end{tabular}

MMS = Min-Max Normalization, MAN = Max-Abs Normalization, B.L. = the stations in the Beiluo River sub-basin.

Five different input filtering strategies are given from S1 to S5. For the scenarios without PCA denoising operation (from S1 to S4), different rainfall and runoff data combinations have been designed. Evaluating the impact of the inclusion of rainfall data, since the $\mathrm{R}$ values and the grey relation grades of the data at meteorological stations are very close (0.094 0.124 and 0.979 0.980, respectively), all the rainfall data from meteorological stations are included in S1 and S3, while excluded in S2 and S4. Also, considering the application of data at similar adjacent regions when using the hydrological analogy method for runoff calculation, to explore whether the similarity would also be valuable to the GRU forecasting model, the runoff data at the stations in the Beiluo River is included in S1 and S2, while excluded in S3 and S4. For the scenarios with PCA denoising operation, S5 and S6 can evaluate the impact of the PCA processed data on the models' performance.

Furthermore, S6 is given to compare with S5 to illustrate the architecture's potential influence when constructing the model. The primary GRU cells have been utilized in S5 and the bi-GRU cells in S6.

Forty-eight different combinations of the hyperparameters have been set for each scenario to evaluate the models' robustness in the model construction process. A summary of the combinations is given in Table 4.

Table 4. A summary of different combinations of the hyperparameters in each scenario.

\begin{tabular}{ccccc}
\hline \multirow{2}{*}{ Num. of Layers } & \multirow{2}{*}{ Input Time Steps } & \multicolumn{3}{c}{ Num. of Hidden Units } \\
\cline { 3 - 5 } & & Layer $\mathbf{1}$ & Layer $\mathbf{2}$ & Layer 3 \\
\hline 1 & \multirow{3}{*}{$5 \sim 10$} & 1 & - & - \\
2 & & $10,20,30$ & 1 & - \\
3 & & $10,20,30,40$ & 10 & 1 \\
\hline
\end{tabular}

Note: The number of hidden units is the number of columns that the weighting matrix has, the larger number is, the stronger ability the model will have to fit the nonlinearity, but the possibility of overfitting may also increase.

The input dataset (from 2007 to 2014) has been divided into the training set, validation set, and testing set according to a ratio of 6:2:2. The validation dataset has been utilized for all of the hyperparameter combinations in accordance with assessing the impact of different inputs and architectures on the model's robustness. For accuracy evaluation, both the performance in the validation set and testing have been considered. Additionally, the training dataset has a small batch size of 10 samples. Five hundred epochs have been executed in the training process.

\subsection{PCA Denoised Data}

The scree plot of the components for the PCA denoising operation is given in Figure 6. According to the scree plot, the explained variance declines dramatically, while the accumulative explained variance increases significantly before the fourth component. Quantitatively, the first four main components explained $91.11 \%$ of the variance. This result indicates that the remaining 20 components have contributed limited information $(8.89 \%)$. Hence, after the PCA denoising operation, four of 24 components 
are maintained and utilized as the input dataset in scenario S5 and S6 (refer to Table 3 in Section 3.2). It is of note that since the PCA denoising operation is a subjective process, the amount of remaining information could still be optimized (if needed) according to the further training and validation process in the modelling stages.

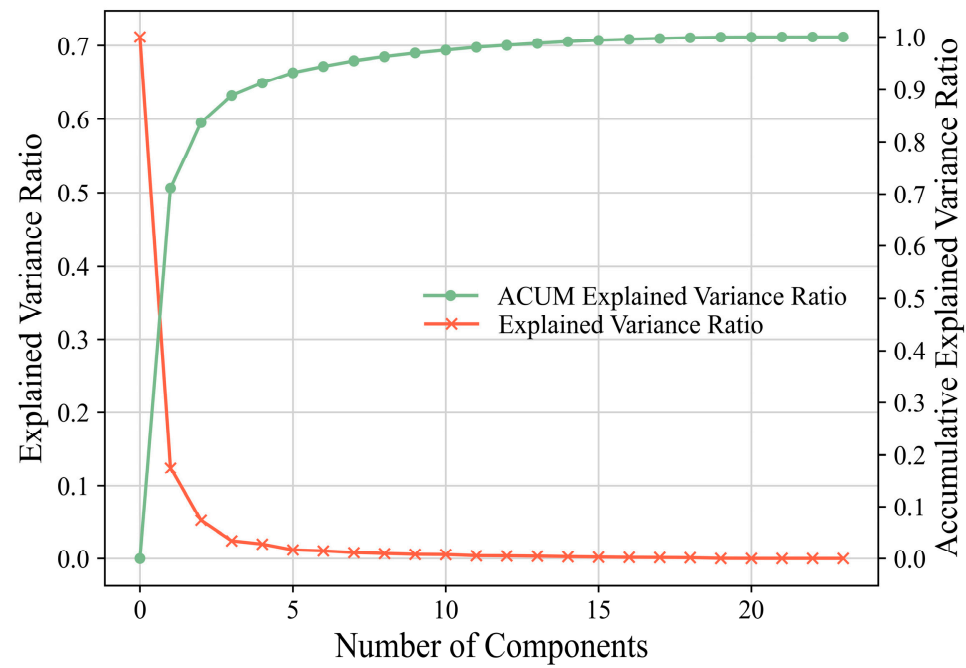

Figure 6. Scree plot of the components, the green line represents the accumulative explained variance ratio, the red line represents the explained variance ratio of each component.

\section{Results and Discussions}

\subsection{Robustness Evaluation}

\subsubsection{Overall Evaluation}

Using the validation set to evaluate the model's robustness to the various hyperparameters, Figure $7 \mathrm{a}-\mathrm{c}$ show the distribution of NSE, RMSE, and MAE, respectively.

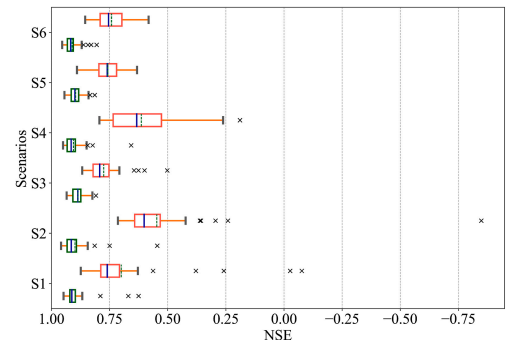

(a)

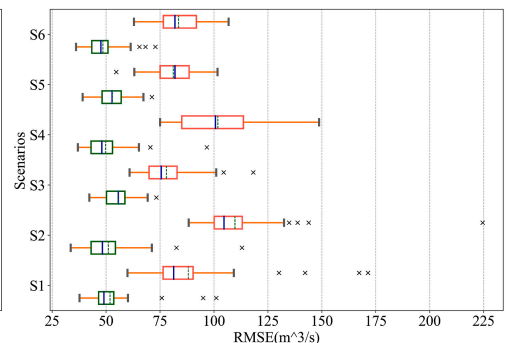

(b)

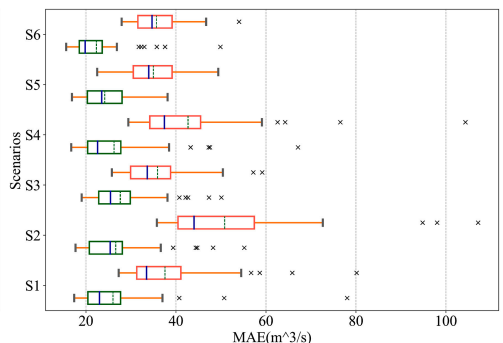

(c)

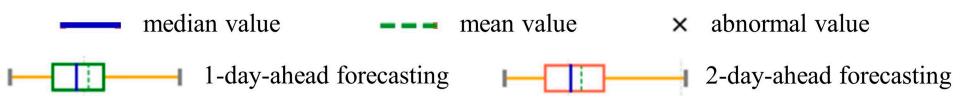

Figure 7. Box plots of the distribution of evaluation metrics in the validation stage. (a) Box plot of the distribution of NSE; (b) box plot of the distribution of RMSE; (c) box plot of the distribution of MAE.

Comparing S1 and S3 with S2 and S4, a lower inter-quartile range (IQR) has been observed in either the distributions of NSE, RMSE, and MAE in both the one-day-ahead $(\mathrm{T}+1)$ and two-day-ahead $(\mathrm{T}+2)$ forecasting. Moreover, this phenomenon is more evident in $\mathrm{T}+2$ forecasting. This result means that the metrics in S1 and S3 are more aggregated than in S2 and S4. Considering the abnormal values, S1 and S3 also tend to have a higher NSE, a lower RMSE, and a lower MAE than S2 and S4 when bad fitting occurs. Additionally, S1 and S3 also have a higher mean value and median value of NSE, lower ones of RMSE, and lower ones of MAE. These results mean that the model with the rainfall data 
is less sensitive and of greater robustness to the various hyperparameters and will require less effort on the hyperparameter optimization.

Comparing S1 and S2 with S3 and S4, though significant difference has not been observed from the aspect of IQR for either of the distributions of metrics in both the $T+1$ and $T+2$ forecasting, the abnormal values indicate that that the inclusion of the runoff data in the adjacent region results in larger deviations when the hyperparameter varies. Thus, though with high numerical relevance, the runoff data in the adjacent region introduces noises and increases the difficulty of the hyperparameter optimization process. Since it is less likely to cause serious deviation, excluding these runoff data can make the model more robust to the hyperparameters.

The metrics' distribution patterns in S5 and S6 in both the T +1 and $\mathrm{T}+2$ forecasting show that the metrics are at least as aggregated as the one in the scenario with the best manual filtered input (S3), indicating that the PCA denoising is an effective input filtering strategy. The PCA denoising operation has significantly improved the model's robustness to the various hyperparameters with a premise of good forecasting performance. Overall, comparing S6 with S5, the bi-directional architecture does not suggest a significant effect on the robustness of the model to various hyperparameters.

\subsubsection{Time Step Standard Deviation of the Evaluation Metrics}

As the length of the time step is the most vital hyperparameter that determines the amount of information in the input, the model's robustness to it should be evaluated independently. Table 5 shows the mean values of the evaluation metrics' standard deviation when the input time step varies. The higher the values are, the less likely the model to have a stable performance on the metrics. Thus, the model will require more effort in time step optimization. In other words, it will be less robust to the length of the input time steps. Similar to patterns obtained from overall evaluation, from the comparison between $\mathrm{S} 1$ and $\mathrm{S} 2$, as well as $\mathrm{S} 3$ and $\mathrm{S} 4$ in either $\mathrm{T}+1$ or $\mathrm{T}+2$ forecasting, the inclusion of rainfall data tends to enhance the time step robustness of the model. Additionally, the enhancement effect has been observed to be more evident in $\mathrm{T}+2$ forecasting. The comparison between $\mathrm{S} 1$ and S3 with S2 and S4, correspondingly, shows that though numerically relevant to the prediction target, the runoff data from the adjacent sub-basin will introduce noise and then hinder the model's time step robustness in the validation stage. Consequently, the time step optimization will be trickier. In comparing S5 and S6, the single directional architecture is more robust than the bi-directional architecture to the time step variations. This phenomenon is different from the overall view and can be explained by the fact that bi-directional architecture is more dependent on the information in the context. When the length of the sequence changes, the amount of information in the context varies.

Table 5. Time step standard deviation of each scenario for one-day-ahead and two-day-ahead forecasting in the validation set.

\begin{tabular}{ccccc}
\hline Prediction Target & Scenario & NSE & RMSE $\left.\mathbf{( m}^{\mathbf{3}} \mathbf{s}\right)$ & MAE $\left(\mathbf{m}^{\mathbf{3}} \mathbf{s}\right)$ \\
\hline \multirow{5}{*}{$\mathrm{T}+1$} & S1 & 0.045 & 9.552 & 7.790 \\
& S2 & 0.046 & 10.225 & 7.355 \\
& S3 & 0.023 & 5.634 & 6.849 \\
& S4 & 0.037 & 8.915 & 8.325 \\
& S5 & 0.023 & 5.713 & 4.693 \\
& S6 & 0.028 & 7.409 & 5.436 \\
\hline \multirow{5}{*}{$\mathrm{T}+2$} & S1 & 0.116 & 15.080 & 7.983 \\
& S2 & 0.148 & 15.370 & 13.799 \\
& S3 & 0.061 & 10.062 & 6.825 \\
& S4 & 0.110 & 14.560 & 10.106 \\
& S5 & 0.057 & 9.825 & 5.762 \\
& S6 & 0.068 & 11.105 & 5.143 \\
\hline
\end{tabular}

$\mathrm{T}+1$ = one-day-ahead forecasting, $\mathrm{T}+2=$ two-day-ahead forecasting. 


\subsection{Accuracy Evaluation}

\subsubsection{Overall Evaluation}

Figures 8 and 9 is the scatter graph and the hydrograph of the best model considering the overall performance in the validation set and testing set for one-day-ahead forecasting and two-day-ahead forecasting. The NSE values in the validation set and the testing set all exceed 0.95 , indicating that the model can provide runoff prediction with high precision. The results also suggest that though the forecasting accuracy declines significantly with the increasing lead time, the NSE values are still over 0.83 , acceptable, and meaningful for practical use. Furthermore, the scatter plots and the hydrographs show that the apparent deviations are more likely to occur as the flowrate increases. This pattern is more evident in the $\mathrm{T}+2$ forecasting, in other words, in the forecasting with a longer lead time. From the aspect of the hydrological mechanism, the large flowrates in this research area are usually caused by intensive rainfall processes, in which the infiltration excess (Horton) runoff's occupancy increases [63]. Since the Horton runoff process driven by intensive rainfalls has a relatively short duration [64], although the runoff data at the distant stations can partially explain the flowrate at the prediction target, it is still difficult for the models to accurately predict the large flowrate two days later. The performance comparison between $\mathrm{T}+1$ and $\mathrm{T}+2$ forecastings also shows that the rainfall and runoff data one day before the prediction date is valuable. This can be explained by the fact that in some rainfall-runoff events, the data's time lags caused by the distance between the prediction target and some adjacent upstream stations are less than two days. Additionally, the deviations when dealing with large flowrates may also be caused by a fact that the weighting matrix does not take the seasonal variation of the rainfall-runoff mechanism [65] into account.
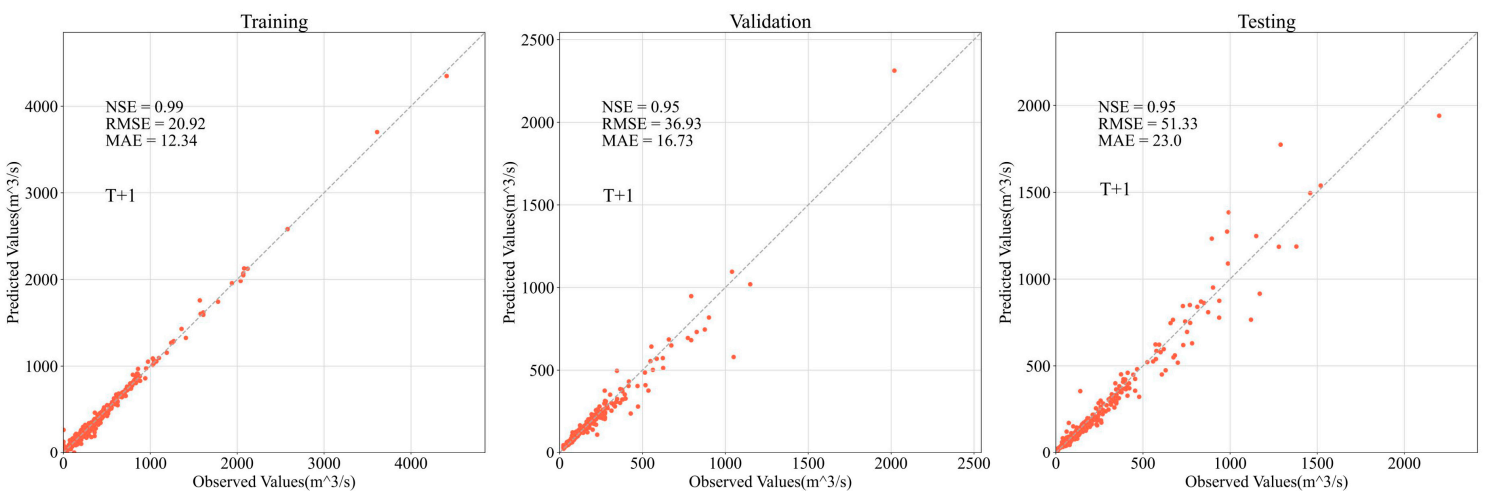

(a)
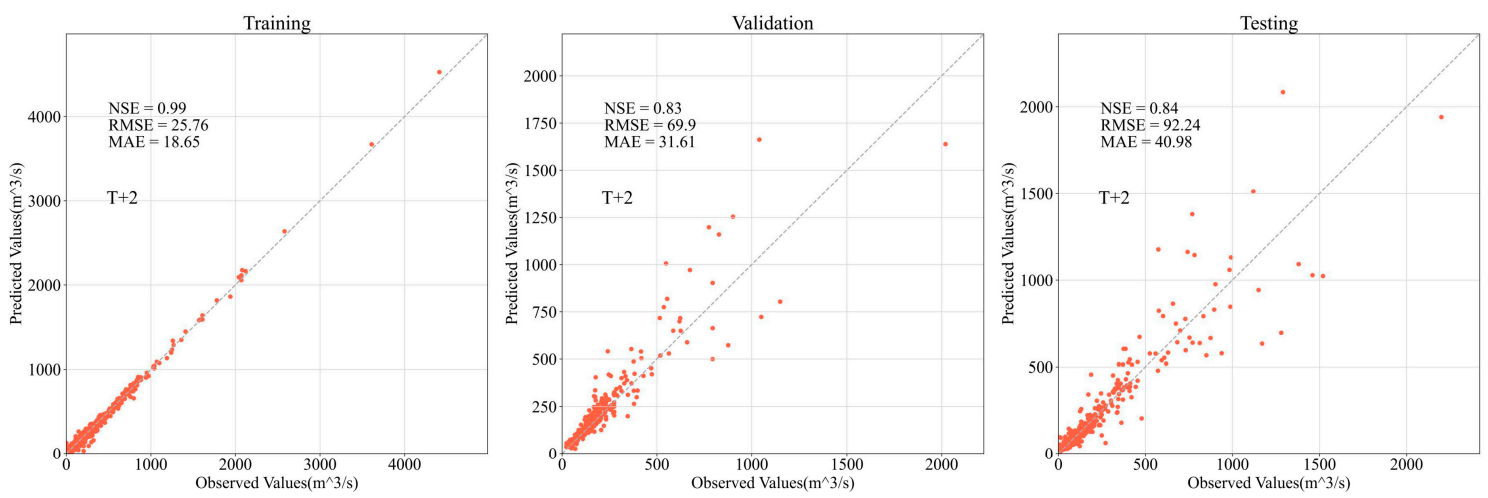

(b)

Figure 8. Scatter plots of the fitting results for the best models. (a) Fitting result of one-day-ahead $(\mathrm{T}+1)$ forecasting in the training, validation, and testing stage; (b) Fitting result of two-day-ahead $(\mathrm{T}+2)$ forecasting in the training, validation, and testing stage. 


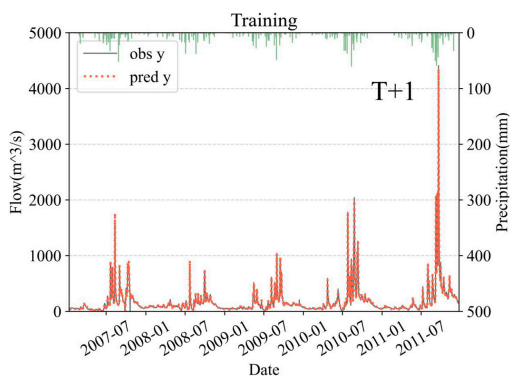

Date

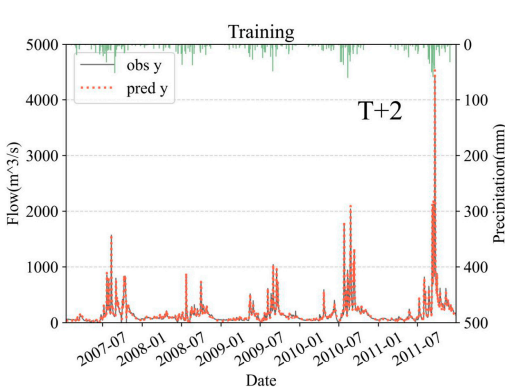

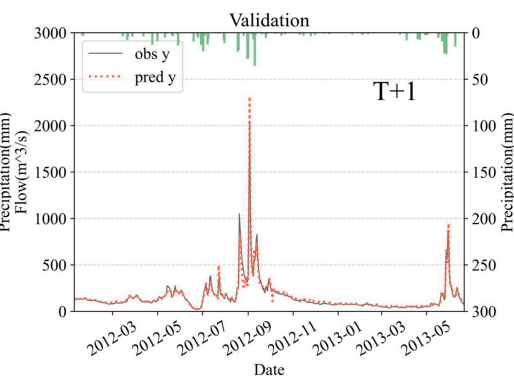

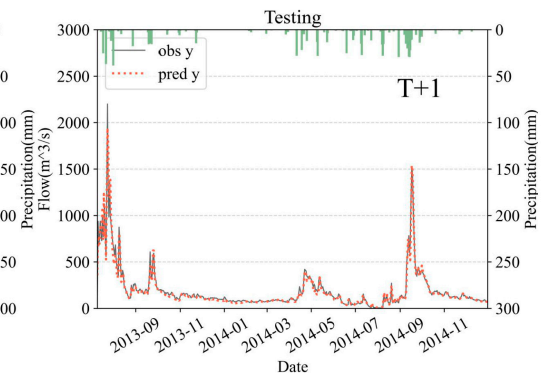

(a)
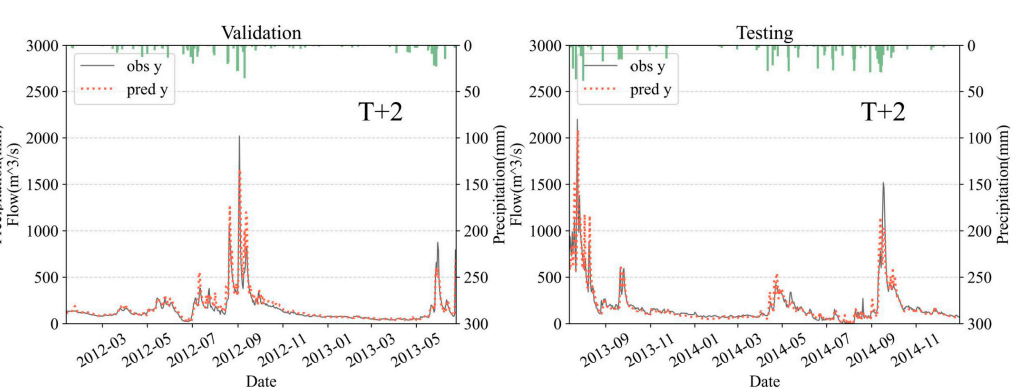

(b)

Figure 9. Hydrographs of the forecasting results for the best models. The green bars represent the rainfall data at the Pucheng meteorological station. (a) Hydrograph of one-day-ahead $(T+1)$ forecasting in the training, validation, and testing stage; (b) Hydrograph of two-day-ahead $(\mathrm{T}+2)$ forecasting in the training, validation, and testing stage.

Table 6 shows the overall evaluation metrics (equivalently considered the results of the validation and testing stage) of the best models in different scenarios; the best model for $\mathrm{T}+1$ forecasting occurs in S4, that is, the scenario without the runoff data in the Beiluo River basin and the rainfall data. In this case, the NSE reaches 0.951 , and the RMSE, MAE, has a value of $44.132 \mathrm{~m}^{3} / \mathrm{s}$ and $19.868 \mathrm{~m}^{3} / \mathrm{s}$, respectively. The NSE values in all the scenarios exceed 0.9 for $\mathrm{T}+1$ forecasting, and in most of the scenarios exceed 0.8 for $\mathrm{T}+2$ forecasting, which means that either of the input filtering strategies can have an acceptable prediction accuracy after the optimization of hyperparameters. However, there are still some differences determining which kind of strategy is better.

Table 6. The overall evaluation metrics of the validation and testing set for the best models in each scenario.

\begin{tabular}{ccccc}
\hline Prediction Target & Scenario & NSE & RMSE $\left(\mathbf{m}^{\mathbf{3}} / \mathbf{s}\right)$ & MAE $\left(\mathbf{m}^{\mathbf{3}} / \mathbf{s}\right)$ \\
\hline \multirow{5}{*}{$\mathrm{T}+1$} & S1 & 0.937 & 50.088 & 24.089 \\
& S2 & 0.945 & 47.449 & 21.276 \\
& S3 & 0.927 & 54.695 & 28.848 \\
& S4 & 0.951 & 44.132 & 19.868 \\
& S5 & 0.941 & 48.402 & 24.178 \\
& S6 & 0.946 & 46.511 & 20.147 \\
\hline \multirow{5}{*}{$\mathrm{T}+2$} & S1 & 0.835 & 82.436 & 38.032 \\
& S2 & 0.745 & 98.346 & 42.062 \\
& S3 & 0.836 & 81.469 & 39.625 \\
& S4 & 0.801 & 89.179 & 37.733 \\
& S5 & 0.828 & 84.130 & 39.014 \\
& S6 & 0.836 & 81.069 & 36.293 \\
\hline
\end{tabular}


For T + 1 forecasting, comparing S1 with S2, as well as S3 with S4, the NSE in S1 and S3 is correspondingly lower than in S2 and S4. Meanwhile, the RMSE and MAE in S1 and S3 are higher than in S2 and S4. This phenomenon indicates that the inclusion of rainfall data decreases the optimized accuracy when forecasting. The reason is that the rainfall data at some distant stations (e.g., Longxian, $296.9 \mathrm{Km}$ to the prediction target) don't explain the runoff at the prediction target 1 day later, but are included in S1 and S3 during the training processes. The impact of the inclusion or exclusion of the runoff data in the Beiluo River basin on the optimized forecasting accuracy is not significant through the comparison between S1 and S3, as well as S2 and S4. The results of S5 and S6 that are two scenarios with PCA denoising show that the models with PCA denoised dataset can provide almost the same level of accuracy (NSE reaches 0.945 and 0.946 in S5 and S6) when comparing the best result of the model (has an NSE of 0.951) with manual filtered dataset. The comparison between S5 and S6 indicates that a bi-directional architecture enhanced the overall prediction accuracy.

For T +2 forecasting, the comparisons between S1 and S2, as well as S3 and S4 (NSE of 0.835 and 0.745 for S1 and S2, 0.836 and 0.801 for S3 and S4) indicating a result that the incorporation of the rainfall data has improved the accuracy of the forecasting results. This result is opposite to the one obtained in one-day-ahead forecasting. It suggests that the rainfall data acts as a noise for the optimized prediction accuracy in one-day-ahead forecasting. However, it is valuable information for the forecasts that have a more extended lead time. This phenomenon can be partly explained by the lag effect in the rainfall-runoff mechanism. Referring to the distances from the meteorological stations to the prediction target given in Table 2 (Section 3.1), almost all the meteorological stations located in the upstream places have a distance of over $100 \mathrm{Km}$ (except for the Pucheng station) to the prediction target. Runoff generated in those places needs time to move to the prediction target. As the result, the rainfall data at those meteorological stations two or more days ago mainly contribute to the flowrate at the prediction target at the forecasting date. These contributions have been recorded in the input time series. Different from the condition in one-day-ahead forecasting, the comparisons between S1 and S3, in addition to S2 and S4, have suggested a significant difference affected by the inclusion or exclusion of the runoff data in the Beiluo River basin. Affected by the noise introduced by the runoff data in the Beiluo River basin, the results in S1 and S2 have lower NSE values (0.835 and 0.745$)$ and higher values of the errors comparing with S3 and S4. The causes of that noise are consequences of the similarities of the runoff data due to the geographic homogeneity between the Beiluo River basin and the prediction target. According to the locations of the hydrological stations, as well as the landscape of the research area (Figure 5), the hydrological stations in the Beiluo River basin are close to the prediction target (Nanronghua, Zhuangtou, and Zhangcunyi have a distance of 23.5, 49.4, and $158.5 \mathrm{Km}$ to the prediction target, respectively), and with a similar underlying surface. For $\mathrm{T}+2$ forecasting, the runoff data at least two days ago near the prediction target would contain little valuable information. S5 and S6 show that the models with PCA denoising can provide accurate forecasting results with NSE values exceeding 0.82 and are comparable to, even better than the best results generated by the models with manual data filtering strategies. The best model occurs in S6, which means that the bi-directional architecture performs better than the single directional architecture for two-day-ahead forecasting after hyperparameter optimisation. The outperformance of the bi-directional architecture has been claimed by many studies $[52,53,60,66]$ in other domains and is still supported by this research when applying to runoff forecasting.

\subsubsection{Accuracy of Flood Peak Forecasts}

The peak flow is usually of the greatest interest when carrying out runoff forecasting since it is crucial to flooding alarming. To evaluate the prediction accuracy of the models on the peak flow, we analyzed the maximum flowrate of the flood events in the validation set and testing set. Table 7 shows the relative error of the forecasting results for the selected peak flows. 
Table 7. The relative error of the simulated flood peaks in each scenario for $\mathrm{T}+1$ and $\mathrm{T}+2$ forecasting.

\begin{tabular}{ccccccccc}
\hline \multirow{2}{*}{ Lead Time } & \multirow{2}{*}{$\begin{array}{c}\text { Case } \\
\text { (Year/Month/Day) }\end{array}$} & $\begin{array}{c}\text { Flow } \\
\left(\mathbf{m}^{\mathbf{3}} / \mathbf{s}\right)\end{array}$ & \multicolumn{7}{c}{ Relative Error (\%) } \\
\cline { 5 - 9 } & & & $\mathbf{S 1}$ & $\mathbf{S 2}$ & $\mathbf{S 3}$ & S4 & S5 & S6 \\
\hline \multirow{4}{*}{$\mathrm{T}+1$} & $2012 / 9 / 3$ & 2020 & 16.75 & 4.12 & 13.94 & 14.48 & 4.30 & 17.95 \\
& $2013 / 7 / 24$ & 2200 & 9.97 & 8.75 & 2.63 & 11.81 & 24.40 & 1.81 \\
& $2014 / 9 / 17$ & 1520 & 6.77 & 7.44 & 31.15 & 1.22 & 9.32 & 7.19 \\
& Mean & - & 11.17 & 6.77 & 15.90 & 9.17 & 12.68 & 8.99 \\
\hline \multirow{3}{*}{$\mathrm{T}+2$} & $2012 / 9 / 3$ & 2020 & 38.85 & 57.00 & 18.58 & 17.83 & 14.66 & 18.89 \\
& $2013 / 7 / 24$ & 2200 & 35.74 & 54.11 & 46.29 & 26.52 & 16.18 & 11.80 \\
& $2014 / 9 / 17$ & 1520 & 19.63 & 31.87 & 11.23 & 33.44 & 33.64 & 32.70 \\
& Mean & - & 31.41 & 47.66 & 25.37 & 25.93 & 21.49 & 21.13 \\
\hline
\end{tabular}

For the $\mathrm{T}+1$ forecasting, the comparisons between the mean relative errors of three selected peak flows in S1 and S2, S3 and S4, suggest that the inclusion of the rainfall data exacerbates the error for flood peak forecasts. Le et al. [15] applied the LSTM network into runoff forecasting in the Da River basin (in Vietnam) also obtained a similar result when evaluating the model's performance on flood peak forecasting. Comparing S1 and S3, S2 and S4, the inclusion of the runoff data in the Beiluo River basin led to smaller errors for the peak flow forecasts. This pattern is slightly different from that in the overall accuracy evaluation, indicating that the adjacent runoff data may not have a significant enhancement effect for the whole forecasting process, but is valuable for the peak flow forecasts with a short lead time. Comparing S5 and S6 to other scenarios, the prediction accuracies of the models with PCA denoising for the peak flows are comparable to the models with manual filtering strategies (relative error ranges in 8.99 12.68\% comparing with 6.77 15.90\%). Comparing S6 to S5, a bi-directional structure tends to enhance the prediction accuracy of the flood peaks.

For the $\mathrm{T}+2$ forecasting, contrary to the situation in the $\mathrm{T}+1$ forecasting, rainfall data significantly reduced the relative error of the flood peak forecasts (the mean relative error of the flood peaks in $\mathrm{S} 1$ and S3 is $31.41 \%$ and $25.37 \%$, while in S2 and S4 is $47.66 \%$ and $25.93 \%$ ). This effect is slightly different from one of the results of the abovementioned relevant study [15] in the Da River basin, Vietnam. The difference is mainly caused by the characteristics of the research areas and flood events. The Da River basin is much smaller than the Wei River basin $\left(52,900 \mathrm{Km}^{2}\right.$ compared with $\left.135,900 \mathrm{Km}^{2}\right)$, the time lag effect caused by the distance would not as evident as that in the Wei River basin. The rainfall in that basin is also more intensive. Additionally, the flood event evaluated in that study has a peak flow of over 10,000 $\mathrm{m}^{3} / \mathrm{s}$, indicating a much shorter duration compared with the one in this study. Thus, the rainfall data in the Da River basin does not explain the flood event well. Hence, significant differences in the characteristic of the research area would partially make the patterns vary. The mean error of the flood peaks in S1 and S2 is correspondingly higher than in S3 and S4, revealing that the inclusion of the runoff data from the Beiluo River basin significantly increased the prediction deviation These phenomena show that, though the rainfall data acts as a noise for $\mathrm{T}+1$ forecasts, it can explain the runoff two days later; some information contained in the runoff data in the adjacent region is valuable for the $\mathrm{T}+1$ forecasts since the similarity to the data at the prediction target but acts as a noise for the T +2 forecasts. The comparison between S5, S6, and other scenarios, suggesting that the models with PCA denoising can have a better performance on the peak flow forecasts when the lead time increases. The PCA has effectively filtered the noise and remained valuable information. Similar to that in the $\mathrm{T}+1$ forecasting, the bi-directional architecture has enhanced the peak flow forecasts' accuracy.

\subsection{Recommendations Based on the Evaluation Results}

Some recommendations for future studies and applications of the GRU runoff forecasting model can be provided according to the evaluation results. Considering both the robustness evaluation and accuracy, for the models with manual vector selection preprocessing process, the rainfall data is recommended to be included as the input, especially in long-lead-time forecasts. To be mentioned, 
this recommendation is mainly based on a premise that the rainfall data is sufficient, however, as the acquisition of rainfall data is difficult in some study areas, the continuous hydrologic modelling [67-69] for design simulation would be a potential supplement. In short-lead-time flood forecasts, it can be considered to be excluded since a negative impact on the optimized prediction accuracy. The runoff data from some adjacent tributaries may be valuable for short-lead-time forecasts for the main channel but is strongly suggested to be excluded when the lead time increases. The PCA denoised dataset can make the model more robust and with equivalent accuracy comparing with the manually filtered dataset, thus, we recommend using the PCA as an alternative preprocessing method. The advantage of the PCA when applying to the preprocessing stage in machine learning is also supported by studies in other realms [56,58,70,71]. Similar to the results of the research about NLP [36,72] and other topics $[53,73,74]$, the bi-directional RNN architecture also tends to provide more accurate results and is recommended to be utilized in the GRU runoff forecasting model.

For further studies, more data filtering strategies can be tested. For instance, the runoff data close to the river's source or the runoff data at the upstream tributaries has a low correlation coefficient. The impact of the inclusion or exclusion of this data will also be valuable for other researchers. The seasonal variation can be considered as an attempt to improve the performance of the model. Furthermore, the type of research area will also affect the found patterns and can be explored in the future.

\section{Conclusions}

This research studied the impact of different input filtering strategies and the model construction strategies on the robustness and prediction accuracy of the GRU model. For the input filtering strategies, the impact of the rainfall data, runoff data at the adjacent region with medium to the high correlation coefficient, and PCA denoising operation has been assessed. For the model construction strategies, we have tested single directional architecture and bi-directional architecture. In summary, the main findings of this study include:

i. Based on the premise that the rainfall data is sufficient, its inclusion can enhance the model's robustness when the hyperparameters vary. Additionally, when the lead time increases, this enhancement effect becomes more pronounced. For optimized accuracy, the rainfall data has a negative impact on the forecasts with a short lead time but is valuable for the forecasts with a longer one in either the overall forecasting process or the flood peak forecasting process. Therefore, the rainfall data is recommended to be included in long-lead-time forecasts.

ii. Though a relative high relevance to the prediction target, the runoff data at the adjacent tributary introduces noise that significantly hinders the robustness of the model and will increase the difficulty of the optimization of hyperparameters. Nevertheless, this runoff data also contains valuable information for the flood peak forecasts with a short lead time and, thus, the exclusion of it should be carefully considered according to the purpose of use. For the forecasts with a more extended lead time, this data acts as noise and should be excluded.

iii. The model uses PCA denoising as the input filtering strategy has comparable robustness to the model that uses well manually filtered data as the input. Thus, it can reduce much effort in the data filtering stage. Meanwhile, the model with PCA denoising operation can provide accurate forecasts, especially for the flood peak forecasts when the lead time increases. Thus, the PCA denoising can be an efficient substitution for the manual input filtering process and is recommended to be considered as an alternative preprocessing method in the future.

iv. Despite a slightly lower time-step robustness, the bi-directional architecture has higher prediction accuracy than the single directional architecture for runoff forecasting, therefore, it is suggested to be utilized.

Though the amount of considered input filtering strategies, as well as the combinations of hyperparameters, in addition to the considered architectures, are still limited, the research has revealed 
some meaningful patterns in defined conditions. It could be a reference for other researchers in the data filtering and model construction stages.

Author Contributions: Conceptualization: Q.W.; methodology: Q.Y.; software: Q.W.; formal analysis: Q.W., Y.L.; resources: X.Y.; data curation: X.Y.; writing_original draft preparation: Q.W.; writing—review and editing: Y.L.; visualization: Y.Z.; supervision: J.Y.; project administration: J.Y.; funding acquisition: J.Y. All authors have read and agreed to the published version of the manuscript.

Funding: This research was funded by the National Natural Science Foundation of China, grant number 51779007, and the National Key Research and Development Program of China, grant number 2016YFC0401308.

Conflicts of Interest: The authors declare no conflict of interest.

\section{References}

1. Narbondo, S.; Gorgoglione, A.; Crisci, M.; Chreties, C. Enhancing Physical Similarity Approach to Predict Runoff in Ungauged Watersheds in Sub-Tropical Regions. Water 2020, 12, 528. [CrossRef]

2. Navas, R.; Alonso, J.; Gorgoglione, A.; Vervoort, R.W. Identifying Climate and Human Impact Trends in Streamflow: A Case Study in Uruguay. Water 2019, 11, 1433. [CrossRef]

3. Nazari-Sharabian, M.; Taheriyoun, M.; Ahmad, S.; Karakouzian, M.; Ahmadi, A. Water Quality Modeling of Mahabad Dam Watershed-Reservoir System under Climate Change Conditions, Using SWAT and System Dynamics. Water 2019, 11, 394. [CrossRef]

4. Kratzert, F.; Klotz, D.; Herrnegger, M.; Sampson, A.K.; Hochreiter, S.; Nearing, G.S. Toward Improved Predictions in Ungauged Basins: Exploiting the Power of Machine Learning. Water Resour. Res. 2019, 55, 11344-11354. [CrossRef]

5. Ahmed, U.; Mumtaz, R.; Anwar, H.; Shah, A.A.; Irfan, R. Efficient Water Quality Prediction Using Supervised Machine Learning. Water 2019, 11, 2210. [CrossRef]

6. Liang, J.; Li, W.; Bradford, S.A.; Šimůnek, J. Physics-Informed Data-Driven Models to Predict Surface Runoff Water Quantity and Quality in Agricultural Fields. Water 2019, 11, 200. [CrossRef]

7. Busico, G.; Colombani, N.; Fronzi, D.; Pellegrini, M.; Tazioli, A.; Mastrocicco, M. Evaluating SWAT model performance, considering different soils data input, to quantify actual and future runoff susceptibility in a highly urbanized basin. J. Environ. Manag. 2020, 266, 110625. [CrossRef]

8. Duan, Y.; Meng, F.; Liu, T.; Huang, Y.; Luo, M.; Xing, W.; De Maeyer, P. Sub-Daily Simulation of Mountain Flood Processes Based on the Modified Soil Water Assessment Tool (SWAT) Model. Int. J. Environ. Res. Public Health 2019, 16, 3118. [CrossRef]

9. Fereidoon, M.; Koch, M.; Brocca, L. Predicting Rainfall and Runoff Through Satellite Soil Moisture Data and SWAT Modelling for a Poorly Gauged Basin in Iran. Water 2019, 11, 594. [CrossRef]

10. Wang, G.; Zhou, M.; Takeuchi, K.; Ishidaira, H. Improved version of BTOPMC model and its application in event-based hydrologic simulations. J. Geogr. Sci. 2007, 17, 73-84. [CrossRef]

11. Peng, Y.; Sun, X.; Zhang, X.; Zhou, H.; Zhang, Z. A Flood Forecasting Model that Considers the Impact of Hydraulic Projects by the Simulations of the Aggregate reservoir's Retaining and Discharging. Water Resour. Manag. 2017, 31, 1031-1045. [CrossRef]

12. Paparrizos, S.; Maris, F. Hydrological simulation of Sperchios River basin in Central Greece using the MIKE SHE model and geographic information systems. Appl. Water Sci. 2017, 7, 591-599. [CrossRef]

13. Xevi, E.; Christiaens, K.; Espino, A.; Sewnandan, W.; Mallants, D.; Sørensen, H.; Feyen, J. Calibration, Validation and Sensitivity Analysis of the MIKE-SHE Model Using the Neuenkirchen Catchment as Case Study. Water Resour. Manag. 1997, 11, 219-242. [CrossRef]

14. Tan, M.L.; Ramli, H.P.; Tam, T.H. Effect of DEM Resolution, Source, Resampling Technique and Area Threshold on SWAT Outputs. Water Resour. Manag. 2018, 32, 4591-4606. [CrossRef]

15. Le, X.H.; Ho, H.V.; Lee, G.; Jung, S. Application of Long Short-Term Memory (LSTM) Neural Network for Flood Forecasting. Water 2019, 11, 1387. [CrossRef]

16. Tikhamarine, Y.; Souag-Gamane, D.; Ahmed, A.N.; Sammen, S.S.; Kisi, O.; Huang, Y.F.; El-Shafie, A. Rainfall-runoff modelling using improved machine learning methods: Harris hawks optimizer vs. particle swarm optimization. J. Hydrol. 2020, 589, 125133. [CrossRef] 
17. Parisouj, P.; Mohebzadeh, H.; Lee, T. Employing Machine Learning Algorithms for Streamflow Prediction: A Case Study of Four River Basins with Different Climatic Zones in the United States. Water Resour. Manag. 2020, 34, 4113-4131. [CrossRef]

18. Thapa, S.; Zhao, Z.; Li, B.; Lu, L.; Fu, D.; Shi, X.; Tang, B.; Qi, H. Snowmelt-Driven Streamflow Prediction Using Machine Learning Techniques (LSTM, NARX, GPR, and SVR). Water 2020, 12, 1743. [CrossRef]

19. Ghorbani, M.A.; Deo, R.C.; Kim, S.; Hasanpour Kashani, M.; Karimi, V.; Izadkhah, M. Development and evaluation of the cascade correlation neural network and the random forest models for river stage and river flow prediction in Australia. Soft Comput. 2020, 24, 12079-12090. [CrossRef]

20. Orellana-Alvear, J.; Celleri, R.; Rollenbeck, R.; Muñoz, P.; Contreras, P.; Bendix, J. Assessment of Native Radar Reflectivity and Radar Rainfall Estimates for Discharge Forecasting in Mountain Catchments with a Random Forest Model. Remote Sens. 2020, 12, 1986. [CrossRef]

21. Song, T.; Ding, W.; Wu, J.; Liu, H.; Zhou, H.; Chu, J. Flash Flood Forecasting Based on Long Short-Term Memory Networks. Water 2020, 12, 109. [CrossRef]

22. Poonia, V.; Tiwari, H.L. Rainfall-runoff modeling for the Hoshangabad Basin of Narmada River using artificial neural network. Arab. J. Geosci. 2020, 13, 1-10. [CrossRef]

23. Ali, S.; Shahbaz, M. Streamflow forecasting by modeling the rainfall-streamflow relationship using artificial neural networks. Model. Earth Syst. Environ. 2020, 6, 1645-1656. [CrossRef]

24. Wagena, M.B.; Goering, D.; Collick, A.S.; Bock, E.; Fuka, D.R.; Buda, A.; Easton, Z.M. Comparison of short-term streamflow forecasting using stochastic time series, neural networks, process-based, and Bayesian models. Environ. Model. Softw. 2020, 126, 104669. [CrossRef]

25. Unnikrishnan, P.; Jothiprakash, V. Hybrid SSA-ARIMA-ANN Model for Forecasting Daily Rainfall. Water Resour. Manag. 2020, 34, 3609-3623. [CrossRef]

26. Saha, A.; Singh, K.N.; Ray, M.; Rathod, S. A hybrid spatio-temporal modelling: An application to space-time rainfall forecasting. Theor. Appl. Climatol. 2020, 1-12. [CrossRef]

27. Ghamariadyan, M.; Imteaz, M.A. A wavelet artificial neural network method for medium-term rainfall prediction in Queensland (Australia) and the comparisons with conventional methods. Int. J. Climatol. 2020, 2020, 1-21. [CrossRef]

28. Tikhamarine, Y.; Malik, A.; Souag-Gamane, D.; Kisi, O. Artificial intelligence models versus empirical equations for modeling monthly reference evapotranspiration. Environ. Sci. Pollut. Res. 2020, 27, 30001-30019. [CrossRef]

29. Ferreira, L.B.; Da Cunha, F.F. New approach to estimate daily reference evapotranspiration based on hourly temperature and relative humidity using machine learning and deep learning. Agric. Water Manag. 2020, 234, 106113. [CrossRef]

30. Kao, I.F.; Zhou, Y.; Chang, L.C.; Chang, F.J. Exploring a Long Short-Term Memory based Encoder-Decoder framework for multi-step-ahead flood forecasting. J. Hydrol. 2020, 583, 124631. [CrossRef]

31. Han, Z.; Dian, Y.; Xia, H.; Zhou, J.; Jian, Y.; Yao, C.; Wang, X.; Li, Y. Comparing Fully Deep Convolutional Neural Networks for Land Cover Classification with High-Spatial-Resolution Gaofen-2 Images. ISPRS Int. J. Geo-Inf. 2020, 9, 478. [CrossRef]

32. Collazos-Huertas, D.F.; Alvarez-Meza, A.M.; Acosta-Medina, C.D.; Castaño-Duque, G.A.; Castellanos-Dominguez, G. CNN-based framework using spatial dropping for enhanced interpretation of neural activity in motor imagery classification. Brain Inform. 2020, 7, 1-13. [CrossRef] [PubMed]

33. Hu, F.; Xia, G.; Member, S. Mining Deep Semantic Representations for Scene Classification of High-Resolution Remote Sensing Imagery. IEEE Trans. Big Data. 2020, 6, 522-536. [CrossRef]

34. Sabour, S.; Frosst, N.; Hinton, G.E. Dynamic routing between capsules. Adv. Neural Inf. Process. Syst. 2017, 30, 3857-3867.

35. Hochreiter, S.; Schmidhuber, J. Long Short-Term Memory. Neural Comput. 1997, 9, 1735-1780. [CrossRef]

36. Jang, B.; Kim, M.; Harerimana, G.; Kang, S.U.; Kim, J.W. Bi-LSTM Model to Increase Accuracy in Text Classification: Combining Word2vec CNN and Attention Mechanism. Appl. Sci. 2020, 10, 5841. [CrossRef]

37. Zhu, Y.; Gao, X.; Zhang, W.; Liu, S.; Zhang, Y. A Bi-Directional LSTM-CNN Model with Attention for Aspect-Level Text Classification. Future Internet 2018, 10, 116. [CrossRef]

38. Jelodar, H.; Wang, Y.; Orji, R.; Huang, H. Deep Sentiment Classification and Topic Discovery on Novel Coronavirus or COVID-19 Online Discussions: NLP Using LSTM Recurrent Neural Network Approach. IEEE J. Biomed. Health Inform. 2020, 24, 1. [CrossRef] 
39. Zou, Q.; Xiong, Q.; Li, Q.; Yi, H.; Yu, Y.; Wu, C. A water quality prediction method based on the multi-time scale bidirectional long short-term memory network. Environ. Sci. Pollut. Res. 2020, 27, 16853-16864. [CrossRef]

40. Liu, D.R.; Lee, S.J.; Huang, Y.; Chiu, C.J. Air pollution forecasting based on attention-based LSTM neural network and ensemble learning. Expert Syst. 2020, 37, 1-16. [CrossRef]

41. Song, C.; Zhang, H. Study on turbidity prediction method of reservoirs based on long short term memory neural network. Ecol. Model. 2020, 432, 109210. [CrossRef]

42. Wang, C.; Qi, Y.; Zhu, G. Deep learning for predicting the occurrence of cardiopulmonary diseases in Nanjing, China. Chemosphere 2020, 257, 127176. [CrossRef] [PubMed]

43. Goluguri, N.V.R.R.; Devi, K.S.; Srinivasan, P. Rice-net: An efficient artificial fish swarm optimization applied deep convolutional neural network model for identifying the Oryza sativa diseases. Neural Comput. Appl. 2020, 1-16. [CrossRef]

44. Cho, K.; Van Merriënboer, B.; Gulcehre, C.; Bahdanau, D.; Bougares, F.; Schwenk, H.; Bengio, Y. Learning phrase representations using RNN encoder-decoder for statistical machine translation. In Proceedings of the Conference on Empirical Methods in Natural Language Processing (EMNLP 2014), Doha, Qatar, 25-29 October 2014; pp. 1724-1734. [CrossRef]

45. Wang, C.; Du, W.; Zhu, Z.; Yue, Z. The real-time big data processing method based on LSTM or GRU for the smart job shop production process. J. Algorithms Comput. Technol. 2020, 14, 1-9. [CrossRef]

46. Gao, S.; Huang, Y.; Zhang, S.; Han, J.; Wang, G.; Zhang, M.; Lin, Q. Short-term runoff prediction with GRU and LSTM networks without requiring time step optimization during sample generation. J. Hydrol. 2020, 589, 125188. [CrossRef]

47. Li, W.; Kiaghadi, A.; Dawson, C. Exploring the best sequence LSTM modeling architecture for flood prediction. Neural Comput. Appl. 2020, 3,1-10. [CrossRef]

48. Wu, Y.; Ding, Y.; Zhu, Y.; Feng, J.; Wang, S. Complexity to Forecast Flood: Problem Definition and Spatiotemporal Attention LSTM Solution. Complexity 2020, 2020, 1-13. [CrossRef]

49. Chen, X.; Huang, J.; Han, Z.; Gao, H.; Liu, M.; Li, Z.; Liu, X.; Li, Q.; Qi, H.; Huang, Y. The importance of short lag-time in the runoff forecasting model based on long short-term memory. J. Hydrol. 2020, 589, 125359. [CrossRef]

50. Okkan, U.; Kirdemir, U. Towards a hybrid algorithm for the robust calibration of rainfall-runoff models. J. Hydroinform. 2020, 22, 876-899. [CrossRef]

51. Li, W.; Kiaghadi, A.; Dawson, C. High temporal resolution rainfall-runoff modeling using long-shortterm-memory (LSTM) networks. Neural Comput. Appl. 2020, 6. [CrossRef]

52. Shahid, F.; Zameer, A.; Muneeb, M. Predictions for COVID-19 with deep learning models of LSTM, GRU and Bi-LSTM. Chaos Solitons Fractals 2020, 140, 110212. [CrossRef] [PubMed]

53. Atef, S.; Eltawil, A.B. Assessment of stacked unidirectional and bidirectional long short-term memory networks for electricity load forecasting. Electr. Power Syst. Res. 2020, 187, 106489. [CrossRef]

54. Yang, T.; Zhang, Q.; Wan, X.; Li, X.; Wang, Y.; Wang, W. Comprehensive ecological risk assessment for semi-arid basin based on conceptual model of risk response and improved TOPSIS model-a case study of Wei River Basin, China. Sci. Total Environ. 2020, 719, 137502. [CrossRef] [PubMed]

55. Tang, S.; Jiang, J.; Zheng, Y.; Hong, Y.; Chung, E.S.; Shamseldin, A.Y.; Wei, Y.; Wang, X. Robustness analysis of storm water quality modelling with LID infrastructures from natural event-based field monitoring. Sci. Total Environ. 2021, 753, 142007. [CrossRef] [PubMed]

56. Geng, D.; Zhang, H.; Wu, H. Short-Term Wind Speed Prediction Based on Principal Component Analysis and LSTM. Appl. Sci. 2020, 10, 4416. [CrossRef]

57. Bhagat, S.K.; Tiyasha, T.; Awadh, S.M.; Tung, T.M.; Jawad, A.H.; Yaseen, Z.M. Prediction of sediment heavy metal at the Australian Bays using newly developed hybrid artificial intelligence models. Environ. Pollut. 2021, 268, 115663. [CrossRef]

58. Arozi, M.; Caesarendra, W.; Ariyanto, M.; Munadi, M.; Setiawan, J.D.; Glowacz, A. Pattern Recognition of Single-Channel sEMG Signal Using PCA and ANN Method to Classify Nine Hand Movements. Symmetry 2020, 12, 541. [CrossRef]

59. Huang, C.J.; Shen, Y.; Chen, Y.H.; Chen, H.C. A novel hybrid deep neural network model for short-term electricity price forecasting. Int. J. Energy Res. 2020, 1-22. [CrossRef] 
60. Crisóstomo de Castro Filho, H.; Abílio de Carvalho Júnior, O.; Ferreira de Carvalho, O.L.; Pozzobon de Bem, P.; dos Santos de Moura, R.; Olino de Albuquerque, A.; Rosa Silva, C.; Guimarães Ferreira, P.H.; Fontes Guimarães, R.; Trancoso Gomes, R.A. Rice crop detection using LSTM, Bi-LSTM, and machine learning models from Sentinel-1 time series. Remote Sens. 2020, 12, 2655. [CrossRef]

61. Duan, L.; Wang, W.; Sun, Y.; Zhang, C.; Sun, Y. Hydrogeochemical Characteristics and Health Effects of Iodine in Groundwater in Wei River Basin. Expo. Health 2020, 12, 369-383. [CrossRef]

62. Zeng, G.; Jiang, R.; Huang, G.; Xu, M.; Li, J. Optimization of wastewater treatment alternative selection by hierarchy grey relational analysis. J. Environ. Manag. 2007, 82, 250-259. [CrossRef] [PubMed]

63. Peng, D.; Xu, Z.; Qiu, L.; Zhao, W. Distributed rainfall-runoff simulation for an unclosed river basin with complex river system: A case study of lower reach of the Wei River, China. J. Flood Risk Manag. 2016, 9, 169-177. [CrossRef]

64. Müller, E.N.; van Schaik, L.; Blume, T.; Bronstert, A.; Carus, J.; Fleckenstein, J.H.; Fohrer, N.; Gerke, H.H.; Graeff, T.; Hesse, C.; et al. Herausforderungen der ökohydrologischen Forschung in Deutschland. Hydrol. Wasserbewirtsch. 2014, 58, 221-240. [CrossRef]

65. Deb, P.; Kiem, A.S.; Willgoose, G. Mechanisms influencing non-stationarity in rainfall-runoff relationships in southeast Australia. J. Hydrol. 2019, 571, 749-764. [CrossRef]

66. Chen, X.; He, J.; Wu, X.; Yan, W.; Wei, W. Sleep staging by bidirectional long short-term memory convolution neural network. Futur. Gener. Comput. Syst. 2020, 109, 188-196. [CrossRef]

67. Grimaldi, S.; Nardi, F.; Piscopia, R.; Petroselli, A.; Apollonio, C. Continuous hydrologic modelling for design simulation in small and ungauged basins: A step forward and some tests for its practical use. J. Hydrol. 2020, 125664. [CrossRef]

68. Petroselli, A.; Grimaldi, S. Design hydrograph estimation in small and fully ungauged basins: A preliminary assessment of the EBA4SUB framework. J. Flood Risk Manag. 2018, 11, S197-S210. [CrossRef]

69. Piscopia, R.; Petroselli, A.; Grimaldi, S. A software package for predicting design-flood hydrographs in small and ungauged basins. J. Agric. Eng. 2015, 46, 74-84. [CrossRef]

70. Nguyen, M.D.; Pham, B.T.; Ho, L.S.; Ly, H.B.; Le, T.T.; Qi, C.; Le, V.M.; Le, L.M.; Prakash, I.; Son, L.H.; et al. Soft-computing techniques for prediction of soils consolidation coefficient. Catena 2020, 195, 104802. [CrossRef]

71. Canchala, T.; Alfonso-Morales, W.; Carvajal-Escobar, Y.; Cerón, W.L.; Caicedo-Bravo, E. Monthly rainfall anomalies forecasting for southwestern Colombia using artificial neural networks approaches. Water 2020, 12, 2628. [CrossRef]

72. Premjith, B.; Kumar, M.A.; Soman, K.P. Neural Machine Translation System for English to Indian Language Translation Using MTIL Parallel Corpus. J. Intell. Syst. 2019, 28, 387-398. [CrossRef]

73. Shahmohammadi, H.; Dezfoulian, M.H.; Mansoorizadeh, M. Paraphrase detection using LSTM networks and handcrafted features. Multimed. Tools Appl. 2020, 1-14. [CrossRef]

74. Kwak, G.; Ahn, C.P.H.; Park, K.L.N. Potential of Bidirectional Long Short-Term Memory Networks for Crop Classification with Multitemporal Remote Sensing Images. Korean J. Remote Sens. 2020, 36, 515-525.

Publisher's Note: MDPI stays neutral with regard to jurisdictional claims in published maps and institutional affiliations.

(C) 2020 by the authors. Licensee MDPI, Basel, Switzerland. This article is an open access article distributed under the terms and conditions of the Creative Commons Attribution (CC BY) license (http://creativecommons.org/licenses/by/4.0/). 